\title{
Investigation of In-service Creep Failures of Aircraft Ti-6Al-4V Bolts
}

\author{
Iordanis Spyroglou ${ }^{1}$, Kyriakos Kourousis ${ }^{2, *}$, and Angelos Koutsomichalis ${ }^{3}$ \\ ${ }^{1}$ Electronics Depot Centre-R\&T Centre, Hellenic Air Force, Athens, 16562, Greece \\ ${ }^{2}$ School of Engineering, University of Limerick, Limerick, Ireland \\ ${ }^{3}$ Hellenic Air Force Academy, Laboratory of Materials, TGA 1010, Attica, Greece
}

\begin{abstract}
Three Ti-6Al-4V bolts from mid-life jet aircraft failed during service operation. Each of the failed bolts were installed on a landing gear component. Metallurgical failure analysis indicated that the fracture mechanism is low temperature creep, possibly resulting from a sustained tension load over a long time. Visual inspection revealed ductile fracture morphology with no macroscopic deformation or corrosion. For all bolts fracture occurred close to the root at the first pitches of the thread. Scanning Electron Microscope (SEM) analysis revealed dimple morphologies with micro-void coalescence. Energy Dispersive X-Ray (EDX) analysis did not reveal any material deficiency that would have precipitated failure.
\end{abstract}

\section{Introduction}

Titanium alloys are widely used in aerospace applications since they combine high tensile strengths and low density, resulting in significant weight savings in relation to other high strength alloys e.g. in replacement of high strength low alloy (HSLA) steels used in upper links, struts and truck beams of landing gears [1]. Titanium is an ideal material for applications in aircraft manufacturing with its high strength, low modulus, low density, and excellent corrosion resistance. The low modulus and density in conjunction with the strength enables the use of components (e.g. springs) that have half the volume of steel components with weight reductions of up to $70 \%$ relative to the steel springs $[2,3]$.

Ti-6Al-4V alloy is the most important and widely used titanium alloy in the aerospace industry because of its high strength to weight ratio, good corrosion resistance and attractive mechanical properties. Ti-6Al-4V alloy is a two phase titanium alloy, and usually exhibits a variety of microstructure morphology depending on the chemical composition, processing history and thermal treatment procedures. Its microstructure depends on chemical composition, processing history and thermal treatment procedures and plays an important role in the mechanical properties of alloys, such as strength, ductility, fracture toughness and crack propagation resistance [4,5].

The tensile and fatigue behaviour of the Ti-6Al-4V is highly influenced by its microstructure and the process applied during manufacturing. The thermal cycle governs

* Corresponding author: kyriakos.kourousis@ul.ie 
the distribution of $\alpha$ and $\beta$ phases in the titanium microstructure. Ti-6Al-4V worked above the beta transition temperature $\left(995^{\circ} \mathrm{C}\right)$, shows only $\beta$ phase which is characterized by a cubic centred crystal structure. Under $\beta$ transus temperature, $\alpha$ and $\beta$ phase are present together in the microstructure [6,7].

Bolt and screw joints are widely used in aerospace structures. The ability of a bolt joint to remain tight over a long period of time is occasionally jeopardized due to the creep phenomenon that takes place not only within the softer gasket material but also the flange and bolt materials [8]. In the literature, very few papers address analytically the effect of creep to assist engineers estimate accurately the load relaxation in bolted joints. Creep analyses of bolted flange connections are presented in $[9,10]$. It is well established that the behaviour of bolted joints under operating conditions is primarily influenced by the elastic interaction of the joint members. The complexities associated in prediction of the behaviour of joint in service are due to its statically indeterminate nature and dependence on a large number of design variables [11]. For high temperature applications, the dependence of elastic properties on temperature, thermal expansion along with the time dependent creep behaviour of components play a crucial role in relaxation of gasket stresses with time [12].

The objective of this paper is the failure analysis of three Ti-6Al-4V bolts fitted on different jet aircraft. The paper focuses on the metallurgical analysis of the failed bolts and the fractographic findings to identify the failure cause.

\section{Investigation}

Three fractured Ti-6Al-4V bolts of three approximately 20 -year-old jet aircraft were submitted for examination. These failures occurred within a short interval, approximately three years, after a major maintenance and upgrade visit in an aircraft maintenance organisation. As part of this maintenance visit, the aircraft nose and main landing gear was removed and reinstalled. Each of the ruptured bolts was attached to the rotary selector valve of the nose landing gear, as illustrated in Figure 1.
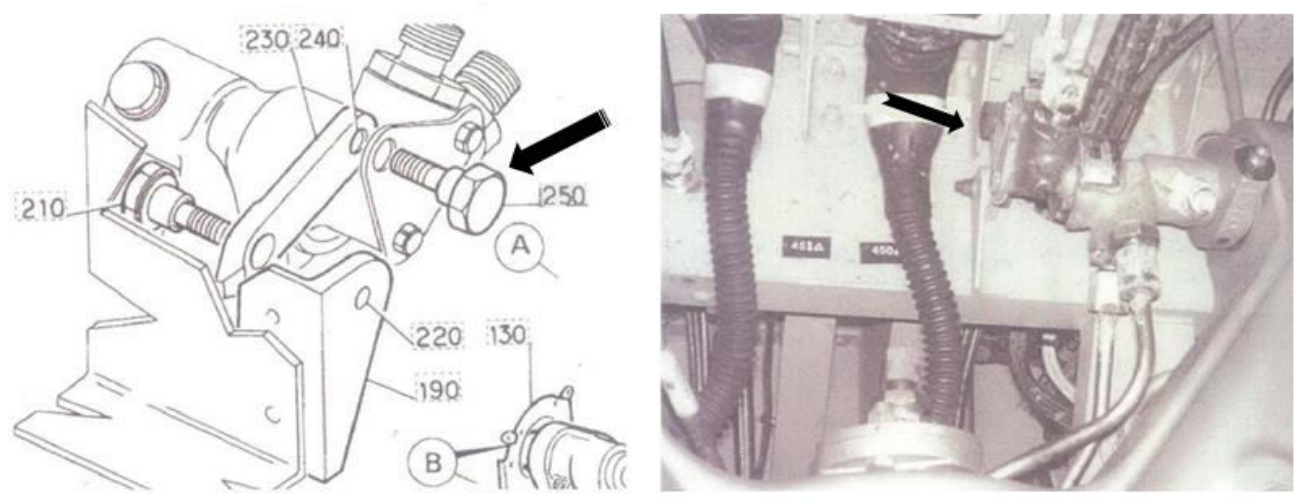

Fig 1. Position of the bolts (indicated with an arrow) on the aircraft landing gear component.

Detailed analysis included visual examination, optical and scanning electron microscopy (SEM) and chemical analysis. The fractured area and the damaged sections of the bolts were cleaned ultrasonically and were examined by stereoscope and SEM.

Samples were cut from the bolts and were prepared following standard metallographic practice and etched using a chemical reagent for microstructural examination. Kellers agent was used for etching the bolt parts. 


\section{Results \& Discussion}

\subsection{Visual inspection}

The three fractured bolts are depicted in Figure 2. Visual examination revealed that the positions of the fracture are all rather close to the root of the bolt thread, which means that the bolts fractured in the vicinity of the last engaged root. This fracture location may be attributed to high stress concentration and severe loading occurring in the region, as also seen in other aircraft fractured bolts [13,14]. Little visible evidence of changes in the thread service condition (e.g. mechanical damage or thread compounds) was discernible.

The fractured surfaces in all cases demonstrate the same fractographic characteristics (Figs 3-5), without indicating any signs of macroscopic deformation or corrosion, while the fracture has occurred at the same point (first active pitch of the thread). The macroscopic fracture characteristics of the three screws are similar: (1) the centre of the bolt fracture appears uniformly coloured, and some axial cracks on it are similar to void; (2) a large number of reflective facets (reflective facet) are shown, and there is no reflective facet around the fracture edge; (3) no macroscopic plastic deformation was visible. The colour of the \#3 screw fracture centre is darker compared with the \#1 and \#2 fractures, and there are also a large number of reflective facets as well as many non-reflective facets around the fracture edge.
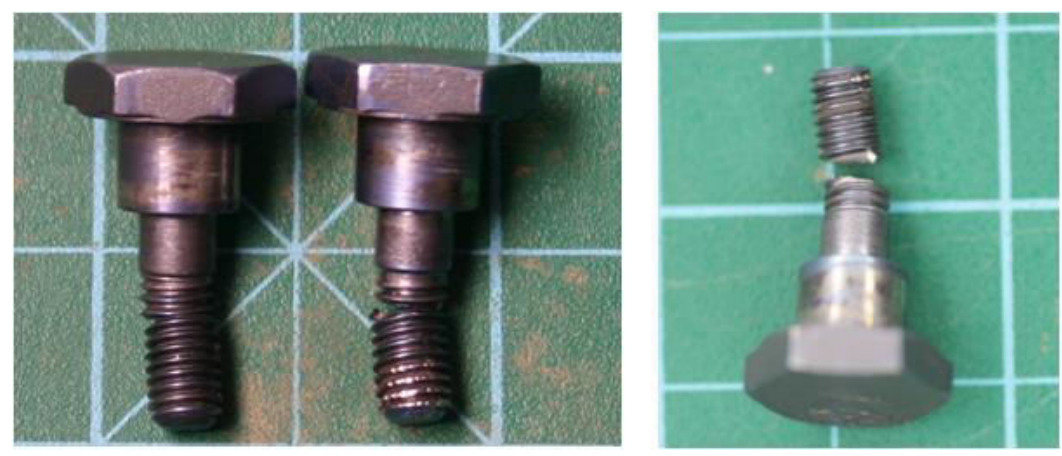

Fig 2. Stereoscopic views of the fractured bolts.

No macroscopic deformation was observed. It is noted that the wear marks observed on the bolt coming might have been caused during the extraction of the fractured part of the bolt from the aircraft landing gear. 

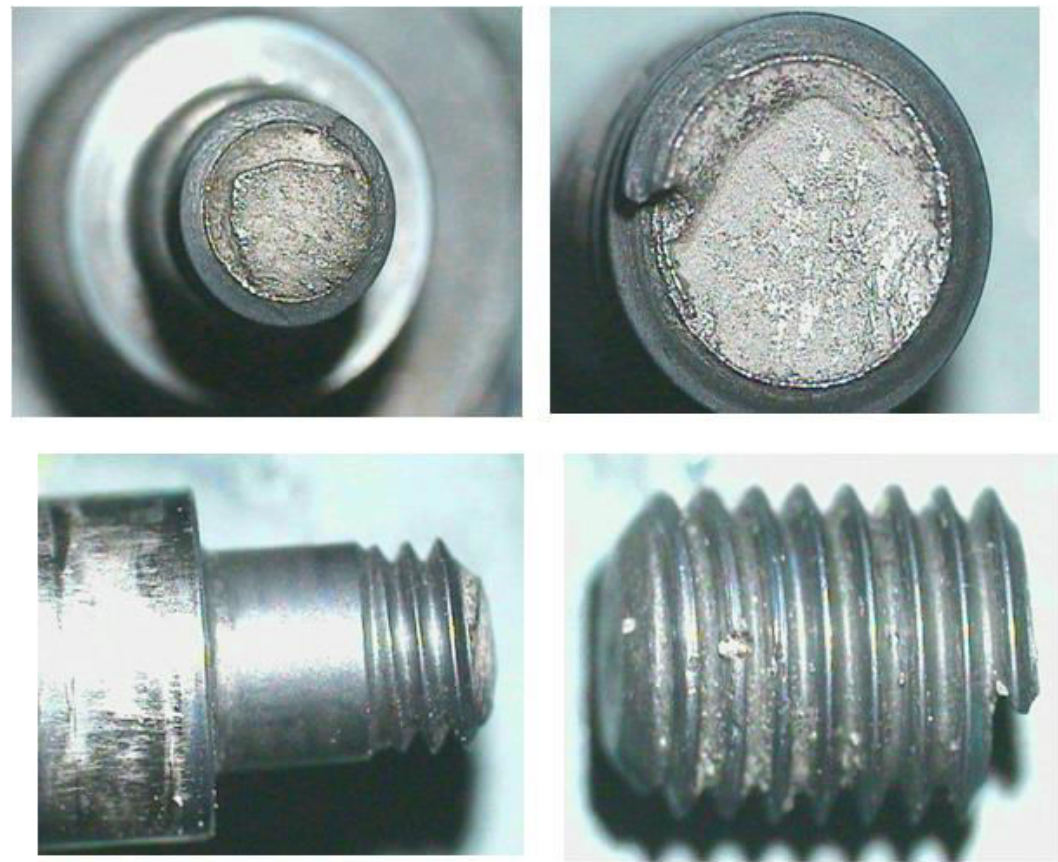

Fig 3. Fracture surface macro appearance and side view of bolt \#1.
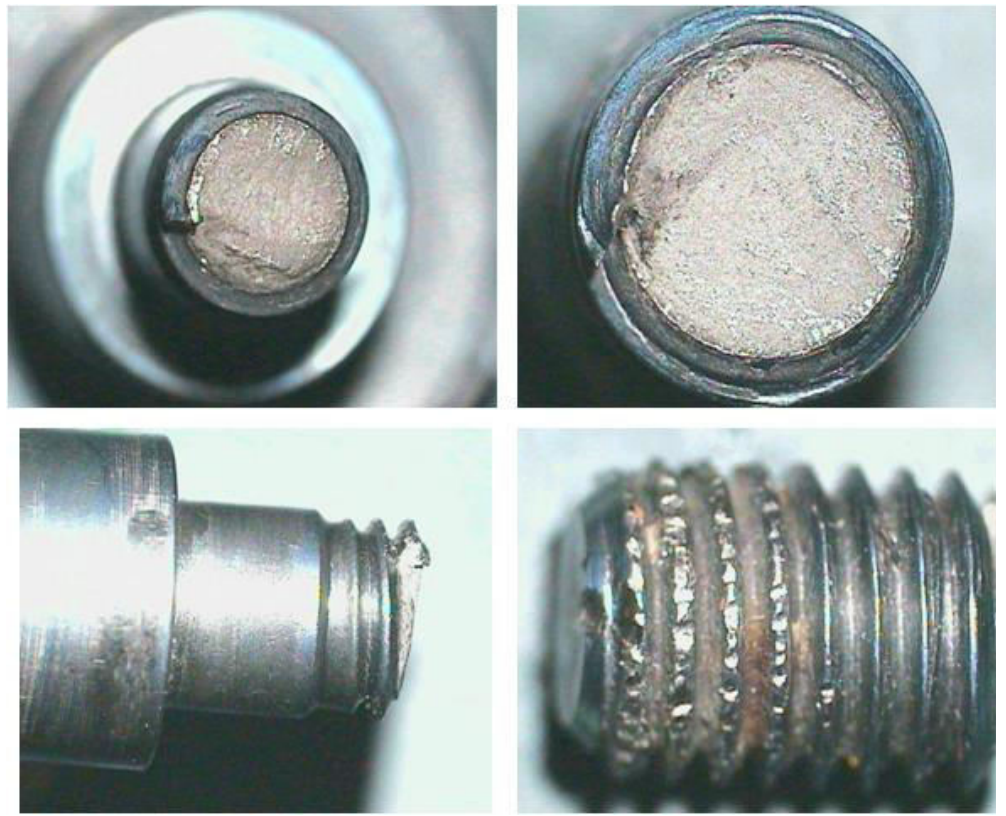

Fig 4. Fracture surface macro appearance and side view of bolt \#2. 

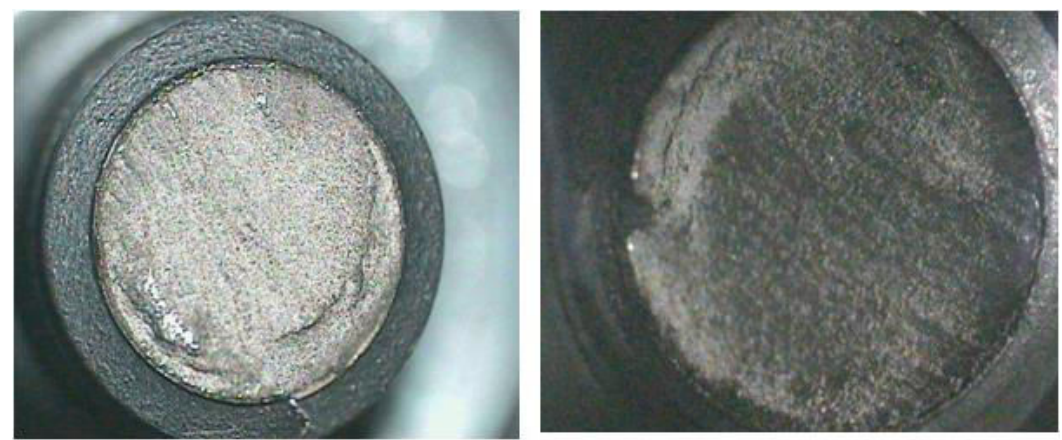

Fig 5. Fracture surface macro appearance of bolt \#3.

\subsection{SEM Examination}

The three fractured bolt specimens were firstly dipped into an acetone solution and cleaned with an ultrasonic washing machine for 5 min prior to their examination with SEM. The micro-fractographic examination conducted with the use of SEM indicated in all cases a clear ductile fracture (cell type surface structure - dimples). The relevant observations are depicted in Figs. 6a and 6b. The Energy Dispersive X-Ray (EDX) analysis confirmed that the chemical analysis of the bolt corresponds to a Ti-6Al-4V alloy (Fig 6c).

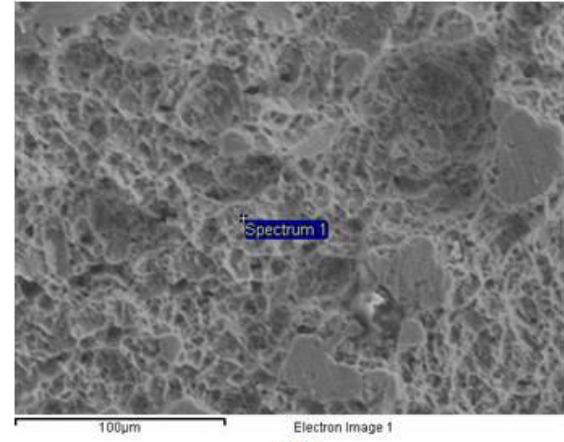

(a)

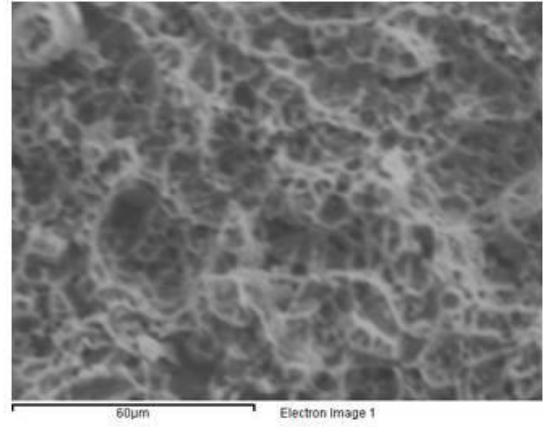

(b)

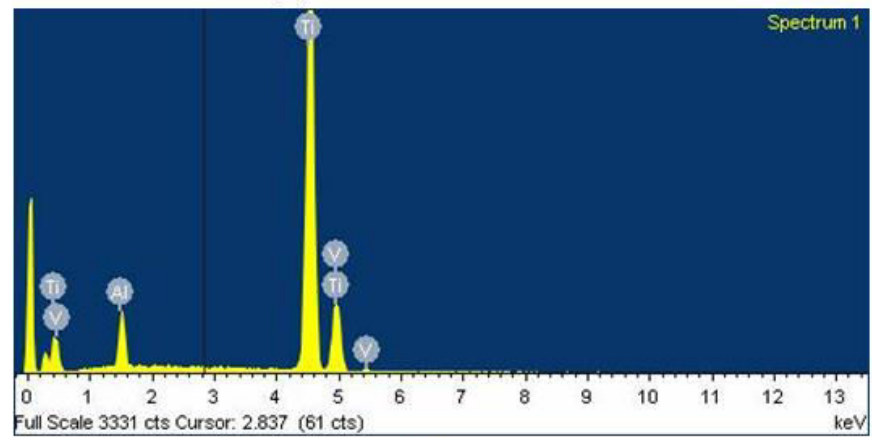

(c)

Fig 6. (a) and (b) SEM images of the fractured screws (c) EDX analysis of the fractured area

Small granular white objects are visible. Morphologies such as dimple can be seen in the margin of the fracture surface. Microvoid coalescence, indicative of a ductile overload was observed as the only evident fracture micro mechanism on all bolts. 
Based on the investigation findings, it is considered possible that the failures are attributed to low temperature creep, possibly resulting from a sustained load (tension) application to the bolts over a prolonged time. This hypothesis is consistent with published scientific and technical studies dealing with low temperature creep performance of Ti-6Al$4 \mathrm{~V}$ alloys $[5,13]$.

In some cases, processing of $\mathrm{Ti}$ alloy bolts is reported to result in the formation of coarse single phase in oxygen rich zones decreasing the ductility and crack propagation resistance and thus leading in fracture $[15,5]$. Also, relevant published reports suggest that the magnitude of the applied load can have significant influence on the manifestation of the creep phenomenon (an overtorque of the bolts may have accelerated strain accumulation). It should be noted that the fractographic characteristics of a creep failure (ductile fracture) are the same as for the case of an overload fracture.

In general, failure in mechanical fasteners and bolts occurs under overload of static (tension, shear, bending or torsion) and dynamic loads. As is often the case in assessing ductile overload failures two different views can be adopted: the first would ascribe the failure to the direct application of a load exceeding the specified strength while the second would ascribe failure to materials properties allowing for plastic deformation [13]. A general form of a bolt torque - tension relationship is [16]:

$$
F_{\text {screw }}=\frac{\left(T-T_{p}\right)}{\left(C_{1} \cdot p+C_{2} \cdot d \cdot \mu_{\text {Thread }}+C_{3} \cdot d_{m} \cdot \mu_{\text {base }}\right)}
$$

where $\mathrm{F}_{\text {screw }}$ is the bolt tension, $\mathrm{T}$ the applied torque at the nut, $\mathrm{T}_{\mathrm{p}}$ is the locking torque, $\mathrm{p}$ is the thread diameter, $d_{m}$ is the nut-base friction diameter and $\mu_{\text {thread }}$ and $\mu_{\text {base }}$ are the thread and nut-base friction coefficients while $\mathrm{C}_{1}$ and $\mathrm{C}_{2}$ are constants related to geometric variables. It is evident from the (1) relationship that the applied torque $\mathrm{T}$ is critical in control of the bolt tension, which should guide further research in identified sources of excessive load application.

At high temperatures creep deformation is associated with the synergistic effect of both microstructural changes and strain accumulation which leads to nucleation of voids, growth and coalescence and subsequently to failure [17]. However, in this case the component under examination (bolts) were not subject to high temperatures, rather than temperatures experienced during normal aircraft operation.

The fact that all failures occurred nearly at the same time and that all affected aircraft had undergone a removal and reinstallation of the nose landing gear, should focus investigation on the maintenance practices applied during the aircraft maintenance visit. This should be able to identify any possible overtorque application during the reinstallation of these bolts.

\section{Conclusions}

1. The failure of three bolts of mid-life aircraft was investigated. Stereoscopic inspection revealed signs of ductile fracture. Little visible evidence of changes in the thread service condition (e.g. mechanical damage or thread compounds) was discernible.

2. The fractured surfaces did not exhibit any signs of macroscopic deformation or corrosion, while the fracture has occurred at the same point (first active pitch of the thread). The macroscopic fracture characteristics of the three bolts exhibited no macroscopic plastic deformation while the centre of the bolt fracture area appeared uniformly coloured with reflective facets.

3. SEM analysis revealed dimple morphologies in the margin of the fracture surface and microvoid coalescence, indicative of a ductile overload thus attributing failure to low 
temperature creep, possibly resulting from a sustained tensile load application to the bolts over a prolonged time.

\section{References}

1. K. Rendigs, Titanium Products Used at AIRBUS, Ti-2003 Science and Technology, G. Lütjering and J. Albrecht, Ed., DGM, Wiley VCH, Weinheim, Germany, 2659-2671 (2004)

2. R. Boyer and R. Briggs, Journal of Materials Engineering and Performance, 22, 29162920 (2013)

3. A. Sherman and S. Seagle, Beta Titanium Alloys in the 1980's, R.R. Boyer and H.W. Rosenberg, eds., AIME, Metallurgical Society of AIME, 281-293 (1984)

4. G. Lutjering, J. Albrecht, O.Ivasishin In: S. Ankem, J. Hall, (eds). Warrendale, The Minerals Metals \& Materials Society, 65-74 (1994)

5. Y. Cui, Q. Meng, B. Zhang, F. Yan, Eng. Fail. Anal. 13, 669-672 (2006)

6. V. Venkateswarlu, D. Tripathy, K. Rajagopal, K. Thomas, T. Venkitakrishnan, Case Studies in Engineering Failure Analysis, 1, 49-60 (2013)

7. L. Gammon, R. Briggs, J. Packard, K. Batson, R. Boyer, and C. Domby, ASM Handbook, Volume 9: Metallography and Microstructures (G.F. Vander Voort, ed.), 899-917 (2004)

8. A. Bouzid, A. ASME Analysis of Bolted Joints PVP 478, 1-7 (2004)

9. R. Bailey, Engineering, 144, 364-365 (1933)

10. J. Matin, J. Franklin Inst., 226, 645-657 (1938)

11. R. Mourya, A. Banerjee, B. Sreedhar, Eng. Fail. Anal. 50, 71-87 (2015)

12. G. Sears, D. King, Int J Press Vessels Pip 81, 513-519 (2004)

13. M. Stevenson, J. McDougall and K. Cline, 34, 75-80 (2003)

14. R. Baggerly, Practical Failure Analysis, 1, 41-46 (2001)

15. Y. Prasad, T. Seshacharyulu, J Mater Process Technol 108, 320-7 (2001)

16. J. Barron, Handbook of Bolts and Bolted Joints, J. Bickford and S. Nassar, eds, Marcel Dekker, 163-178 (1998)

17. S. Baby, B. Kowmudi, C. Omprakash, D. Satyanarayana, K. Balasubramaniamb and V. Kumar, Scripta Materialia 59, 818-821 (2008) 